\title{
Cryoloading: introducing large molecules into live synaptosomes
}

\section{Arup R. Nath, Robert H. C. Chen and Elise F. Stanley*}

Laboratory of Synaptic Transmission, Toronto Western Research Institute, Toronto, ON, Canada

\section{Edited by:}

Dieter Wicher, Max Planck Institute for

Chemical Ecology, Germany

Reviewed by:

Daniela Tropea, Trinity College Dublin,

Ireland

Tetsufumi Ueda, The University of

Michigan, USA

*Correspondence:

Elise F. Stanley, Laboratory of Synaptic

Transmission 7KD-418, Toronto

Western Research Institute, 399

Bathurst Street, Toronto, ON M5T

2S8, Canada

e-mail: elise.f.stanley@gmail.com
Neurons communicate with their target cells primarily by the release of chemical transmitters from presynaptic nerve terminals. The study of CNS presynaptic nerve terminals, isolated as synaptosomes (SSMs) has, however, been hampered by the typical small size of these structures that precludes the introduction of non-membrane permeable test substances such as peptides and drugs. We have developed a method to introduce large alien compounds of at least $150 \mathrm{kDa}$ into functional synaptosomes. Purified synaptosomes are frozen in cryo-preserving buffer containing the alien compound. Upon defrosting, many of the SSMs contain the alien compound presumably admitted by bulk buffer-transfer through the surface membranes that crack and reseal during the freeze/thaw cycle. $\sim 80 \%$ of the cryoloaded synaptosomes were functional and recycled synaptic vesicles (SVs), as assessed by a standard styryl dye uptake assay. Access of the cryoloaded compound into the cytoplasm and biological activity were confirmed by block of depolarization-induced SV recycling with membrane-impermeant

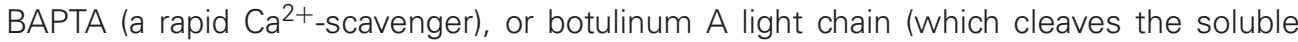
NSF attachment protein receptor (SNARE) protein SNAP25). A major advantage of the method is that loaded frozen synaptosomes can be stored virtually indefinitely for later experimentation. We also demonstrate that individual synaptosome types can be identified by immunostaining of receptors associated with its scab of attached postsynaptic membrane. Thus, cryoloading and scab-staining permits the examination of $\mathrm{SV}$ recycling in identified individual CNS presynaptic nerve terminals.

Keywords: presynaptic, synaptosome, peptide loading, intracellular, synaptic vesicle, recycling, styryl dye

\section{INTRODUCTION}

At a typical synapse in the central nervous system (CNS) a small, $\sim 2 \mu \mathrm{m}$, presynaptic bouton contacts a spine or cell body of a target neuron. Classical transmitters are released by the calcium-gated fusion of synaptic vesicles (SVs) with the surface membrane. The SVs are then recovered, recycled and refilled for reuse. However, the analysis of SV recycling and fusion has been limited by the inability to reliably introduce compounds into the cytoplasm to probe molecular events. Our objective was to develop a method to load CNS presynaptic terminals with test compounds while retaining their ability to exhibit calcium-gated SV recycling.

CNS terminals can be isolated by sub-cellular fractionation as 'synaptosomes' (SSMs), comprising the nerve terminal together with a small scab of postsynaptic membrane, and SV recycling can be monitored by depolarization-induced uptake of green or red styryl dyes, Fei Mao (FM)1-43 or FM4-64, respectively (Bouvier et al., 1996). We introduced compounds into individual identified SSMs by "cryoloading", an idea conceived by combining two published SSM methods: freeze-thaw, to admit the alien compounds (Nichols et al., 1989; Tamura et al., 2001) with cryopreservation, to maintain functionality (Gleitz et al., 1993; Begley et al., 1998). Cryoloading was evaluated in three steps. First, we used imaging to detect uptake of test alien substances of varying size and composition. Second, we used depolarization/extracellular $\mathrm{Ca}^{2+}$. dependent styryl dye uptake to confirm that SV recycling persisted in individual cryoloaded SSMs, and finally, we cryoloaded alien membrane-impermeant compounds that are known to block $\mathrm{Ca}^{2+}$-gated transmitter release to demonstrate utility of the method. In addition, we report a method to identify individual SSM types by their associated postsynaptic receptor types.

\section{METHODS, RESULTS AND DISCUSSION}

To develop an SSM cryoloading protocol we began with methods in several previous reports that have reported retention of functional properties with SSM freeze/thaw and a single report that described SSM loading. Our main challenges were achieving maximal functional SSM retention and loading through the freeze/thaw cycle together while using minimal buffer volumes. The latter is essential for the method to be generally utile since many of the compounds that we had in mind for cryoloading, such as synthetic peptides, bacterial fusion proteins etc., are expensive or in short supply. Clumping, a centrifugation hazard also had to be avoided. While SSMs in clumps were functional, they precluded imaging of individual terminals to monitor styryl dye recycling. The resulting cryoloading method is 
robust; has been used reliably in over 40 experiments to date, and is practical with small quantities of test substance, as illustrated below.

\section{PREPARATION OF FRESH SYNAPTOSOMES}

Fresh SSMs were prepared from 30 whole E15 chick brains as described (Juhaszova et al., 2000; Khanna et al., 2007; Wong et al., 2013). Briefly, the chick brains were first homogenized in homogenization buffer (HB: $0.32 \mathrm{M}$ sucrose; $2 \mathrm{mM}$ EDTA; $10 \mathrm{mM}$ HEPES). After a slow speed spin for $15 \mathrm{~min}$ at $1000 \mathrm{x} g$ (g-max), retaining the supernatant, followed by wash by two high-speed spins for $35 \mathrm{~min}$ at 250,000 $\mathrm{x} \mathrm{g}$, retaining the pellet, the cell membrane/organelle suspension was fractionated on a discontinuous sucrose gradient spun at $100,000 \mathrm{x}$ g. The $0.8 / 1.2 \mathrm{M}$ interface was collected and respun at $20,000 \mathrm{x} \mathrm{g}$ for $30 \mathrm{~min}$. This SSM pellet was resuspended in $2.5 \mathrm{ml}$ Sucrose, EDTA, Tris (SET) buffer (0.32 M sucrose; $1 \mathrm{mM}$ EDTA; $5 \mathrm{mM}$ Tris (adapted from Daniel et al., 2012)), protein concentration was determined by Bradford assay and the SSMs were diluted to $2 \mathrm{mg} / \mathrm{ml}$ (stock SSMs). SSM enrichment has been confirmed extensively by biochemical analysis (Juhaszova et al., 2000; Khanna et al., 2007; Wong et al., 2013). SSMs were plated on cover slips and were fixed and immunostained (methods: Li et al., 2004) with several presynaptic markers including SV2 (Figure 1A), CaV2.2, CSP and SNAP-25 (not shown). SSMs are known to comprise not only the presynaptic terminal but also a variable sized "scab" of the postsynaptic apparatus (Whittaker, 1993; see Figure 2A). We reasoned that the scab would contain residual postsynaptic receptors characteristic for the synapse type and, hence, that these could serve to identify individual SSMs. To our knowledge this has never been done previously. Thus, immunostaining for NMDA or GABA-A receptors identified SSMs with small, but intensely stained SSM-associated patches (Figure 1A) and confirmed that our SSM preparation was heterogeneous and contains both excitatory-glutamatergic and inhibitory-GABAergic terminals.

\section{SYNAPTIC VESICLE RECYCLING FM1-43/4-64 STYRYL DYE ASSAY}

We verified that the fresh SSMs were functional by testing for depolarization $/ \mathrm{Ca}^{2+}$-dependent $\mathrm{SV}$ recycling using the styryl dye uptake method (Figure 1B). SSMs were plated on a coverslip in a $\mathrm{Ca}^{2+}$-containing Krebs-like physiological buffer (KPB: $143 \mathrm{mM} \mathrm{NaCl}, 4.7 \mathrm{mM} \mathrm{KCl}, 1.3 \mathrm{mM} \mathrm{MgSO}_{4}, 1.2$ $\mathrm{mM} \mathrm{CaCl} 2,20 \mathrm{mM}$ HEPES, $0.1 \mathrm{mM} \mathrm{NaH} \mathrm{PO}_{4}, 10 \mathrm{mM}$ glucose; $\mathrm{pH}$ 7.4). The SSMs were then exposed to the same buffer but with high $\mathrm{K}^{+}$(40 mM) with FM1-43 (or FM4- 64; $1 \mu \mathrm{M}$ ) for $2 \mathrm{~min}$ at $30^{\circ} \mathrm{C}$. SSMs were rinsed in $\mathrm{KPB}$ with Advasep-7 to quench residual extracellular dye fluorescence and dye uptake was imaged by fluorescent microscopy (Figure 1B).

\section{SAMPLE OPTIMIZATION FOR FREEZING}

Development of a utile cryoloading method required optimization of several aspects of the SSM sample: the quantity of stock SSMs per sample, the volume of freezing buffer, and centrifugation methods that avoid excessive SSM clumping. We settled on $100 \mu \mathrm{l}$ of the $2 \mathrm{mg} / \mathrm{ml}$ stock for each $50 \mu \mathrm{l}$ cryoloading sample, pelleting the stock at 13,000 RPM (16,000 x g) for $3 \mathrm{~min}$ at $4^{\circ} \mathrm{C}$ (Sorval Hereaus Fresco Tabletop Centrifuge).

\section{SYNAPTOSOME FREEZE-THAW}

Each stock pellet was resuspended in $50 \mu \mathrm{l}$ of cryoloading buffer (CB; 5\% V/V DMSO in SET buffer) and maintained for $3 \mathrm{~min}$ at room temperature $\left(\sim 22^{\circ} \mathrm{C}\right)$. The target loading compounds were dissolved or dispersed in $\mathrm{CB}$ at a high concentration, in volumes ranging from 1 to $6 \mu \mathrm{l}$, and were added to the SSM suspension immediately prior to freezing. We compared two freezing methods. For "rapid-freeze" we immersed the tube in dry ice-cooled isopropanol, and for "slow-freeze" the tube was sealed in a Styrofoam container with parafilm (Sigma-Aldrich) before placing the container in a $-80^{\circ} \mathrm{C}$ freezer. While both methods could be used, the slow-freeze method was favored because rapid-freeze resulted in a high degree of clumping of the thawed SSMs.

We also tested two methods of thawing: "rapid-thaw" with the sealed tubes in a standard cell culture incubator at $37^{\circ} \mathrm{C}$ for $2 \mathrm{~min}$, and "slow-thaw" by incubating the tubes at $4^{\circ} \mathrm{C}$ (Nichols et al., 1989). SSM survival was invariably better with the rapid-thaw method. The thaw process was terminated by addition of $100 \mu \mathrm{l}$ ice cold SET buffer to the sample. Reconstituted SSMs were then pelleted by a $2 \mathrm{~min}$ spin as above. SSMs that had been subjected to the freeze-thaw cycle were indistinguishable from fresh terminals by eye under the light microscope.

\section{ALIEN COMPOUNDS WERE CRYOLOADED INTO THE SYNAPTOSOMES}

We used inert fluorescent dextran (3 or $10 \mathrm{kDa}$; Invitrogen) as the initial cryoloading test compounds. The intensity of SSM staining varied with the concentration of the marker (Figure 1C), consistent with bulk uptake of extracellular medium. To identify the intracellular destination of cryoloaded substances, we froze SSMs in the presence of $10 \mathrm{~nm}$ colloidal gold (EY Laboratories). The SSMs were pelleted, fixed, stained with $2 \%$ osmium tetroxide in $0.1 \mathrm{M}$ cacodylate buffer for $1 \mathrm{~h}$, en bloc stained with $1 \%$ uranyl acetate in water for $1 \mathrm{~h}$, dehydrated and then embedded, thin sectioned and mounted by standard methods. Sections were imaged on a Hitachi HT7000 transmission electron microscope operating at $75 \mathrm{kV}$. After the freeze-thaw cycle SSMs were morphologically intact with dense clusters of SVs and active zones that generally retained an attached postsynaptic fragment (Figure 2A), as described (Whittaker, 1993). Gold particles observed within SSMs were located in the cytoplasm $(N=3$ terminals; Figure $2 \mathrm{~A}$, right panel).

\section{CRYOLOADED SYNAPTOSOMES WERE FUNCTIONAL AND CAPABLE OF VESICLE CYCLING}

The styryl dye uptake method was used to test if cryoloaded SSMs were functional (Figures 2B, 3A). We froze SSMs in the presence of $3 \mathrm{kDa}$-Fluorescein isothiocyanate (FITC) or $10 \mathrm{kDa}-\mathrm{AF} 568$ dextran to tag the cryoloaded sub-population, using a complimentary FM dye for SV recycling assay (FM4-64 and FM1-43, respectively; Figures 2B, 3A). No dye uptake was observed without both $\mathrm{Ca}^{2+}$ in the extracellular medium 

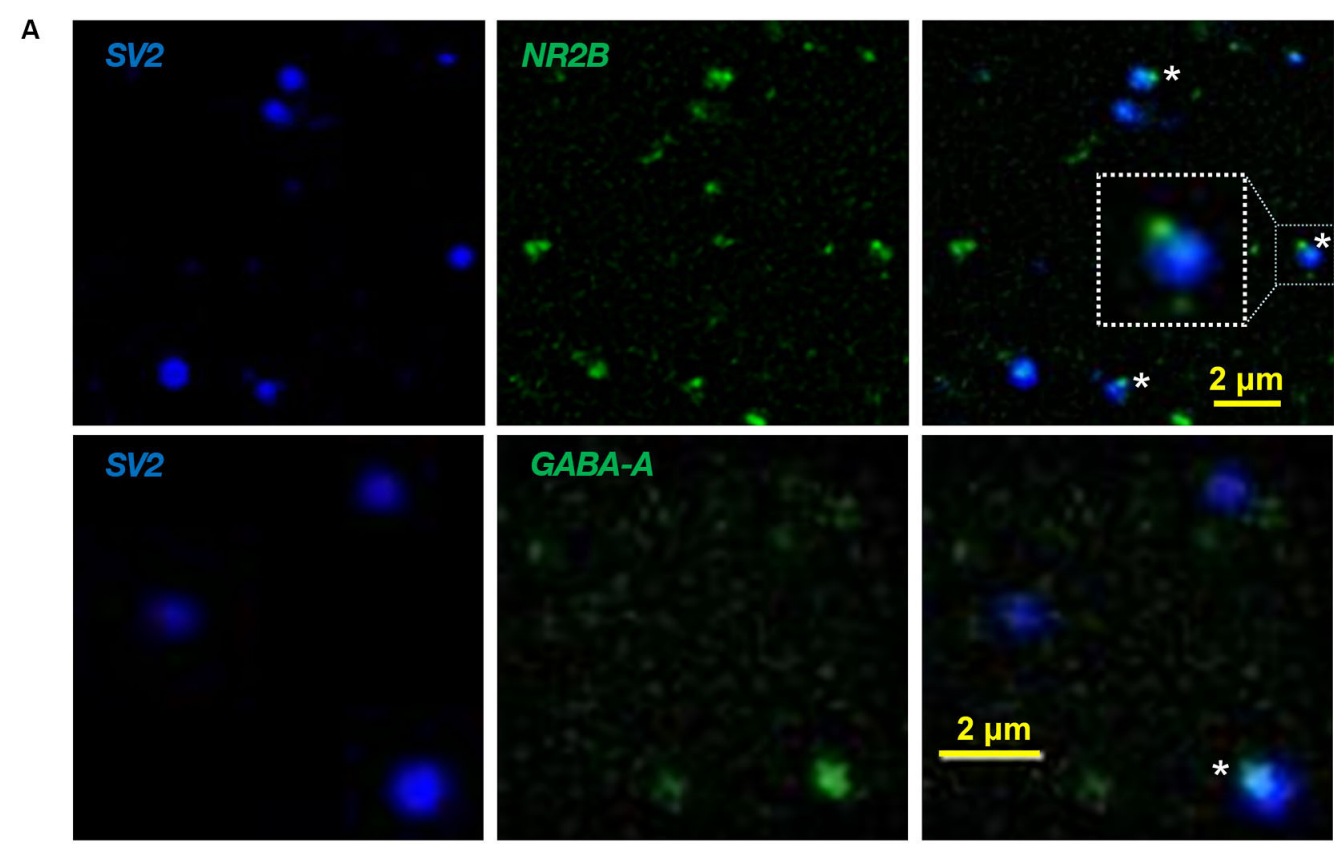

B
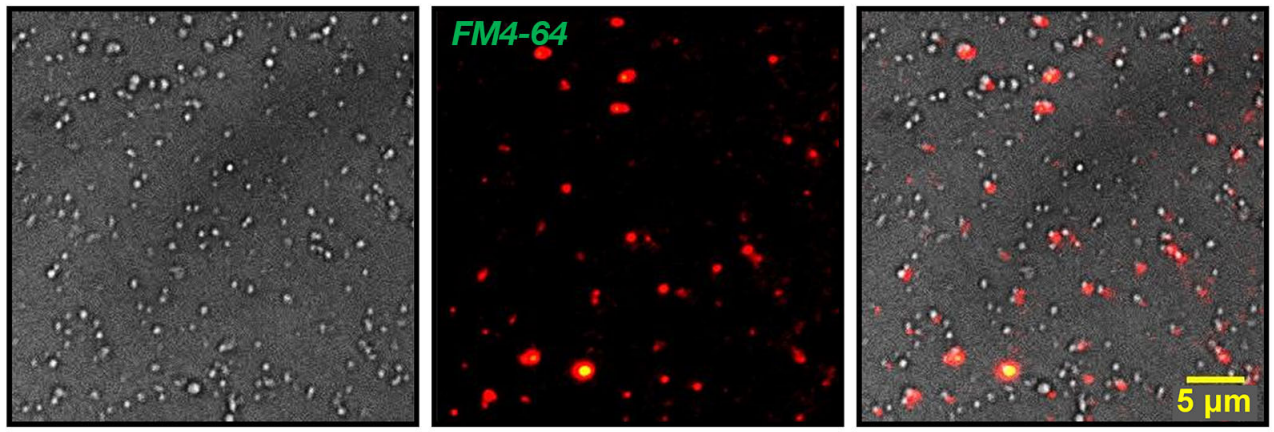

$20 \mu \mathrm{M}$

C

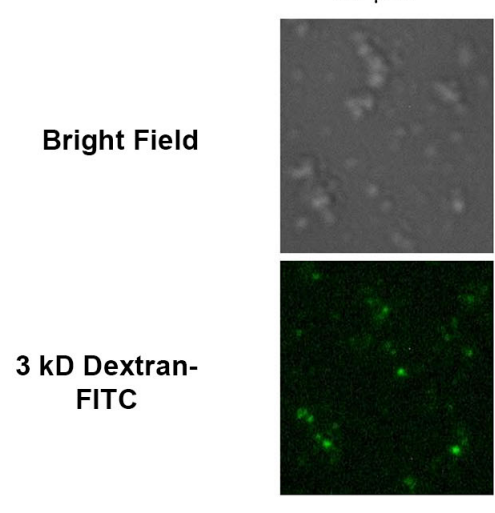

FIGURE 1 | Cryoloading introduces alien compounds into the nerve terminal. (A) Fresh chick brain synaptosomes were plated on coverslips, fixed and immunostained for SV2 (DSHB, 1:1), a SV marker, and either the NMDA receptor subunit, NR2B (Abcam, ab65783; 1:200; upper panel) or GABA-A $\alpha 1$ receptor (Millipore 06-868 1:200, lower panel), as labeled. Staining overlays are shown on the right. SV2 stains SVs selectively and fills the presynaptic terminal. NMDA and GABA-A receptor antibodies identify small patches on the SSM surface $\left({ }^{*}\right.$, enlarged inset), marking the scab of postsynaptic membrane (see also Figure 2A). Light microscopy was carried out on a Zeiss Axioplan upright microscope with a $63 \mathrm{X}$ oil
$10 \mu \mathrm{M}$

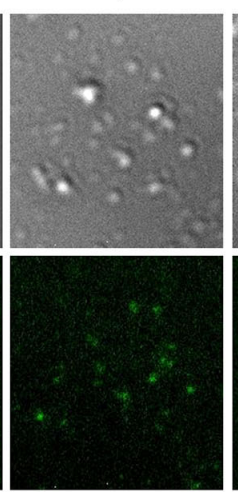

$5 \mu \mathrm{M}$

$2.5 \mu \mathrm{M}$
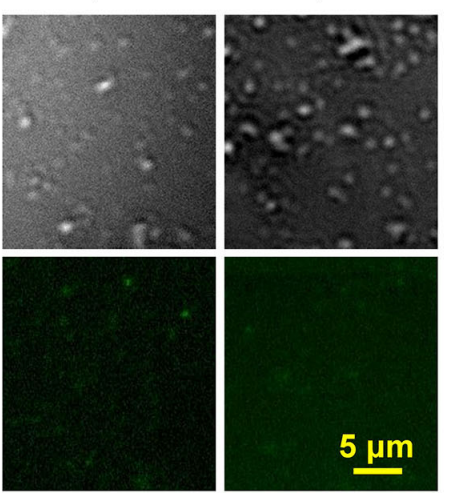

immersion, 1.45 NA objective. Image stacks used for fixed samples, as in A, were deblurred by iterative deconvolution using the Zeiss turnkey software, as described (Li et al., 2004). (B) Nomarski bright field (left), FM4-64 uptake (2 $\mathrm{mM} \mathrm{Ca}^{2+} / 40 \mathrm{mM} \mathrm{K}^{+}$; center) and overlay (right) identifying terminals with functional SV recycling in the SSM fraction. FM dye stained SSM images were background corrected using the Zeiss software function. (C) Chick synaptosomes cryoloaded with $3 \mathrm{kDa}$ dextran-FITC with the marker at the indicated concentrations in the freezing buffer imaged by bright field (upper panel) and fluorescence microscopy (lower panel). 
A

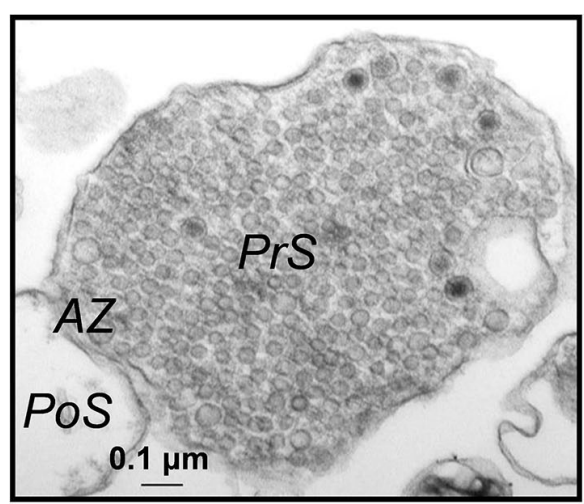

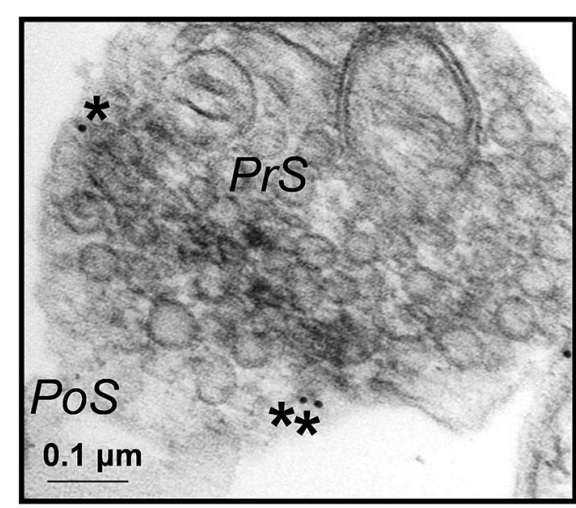

B
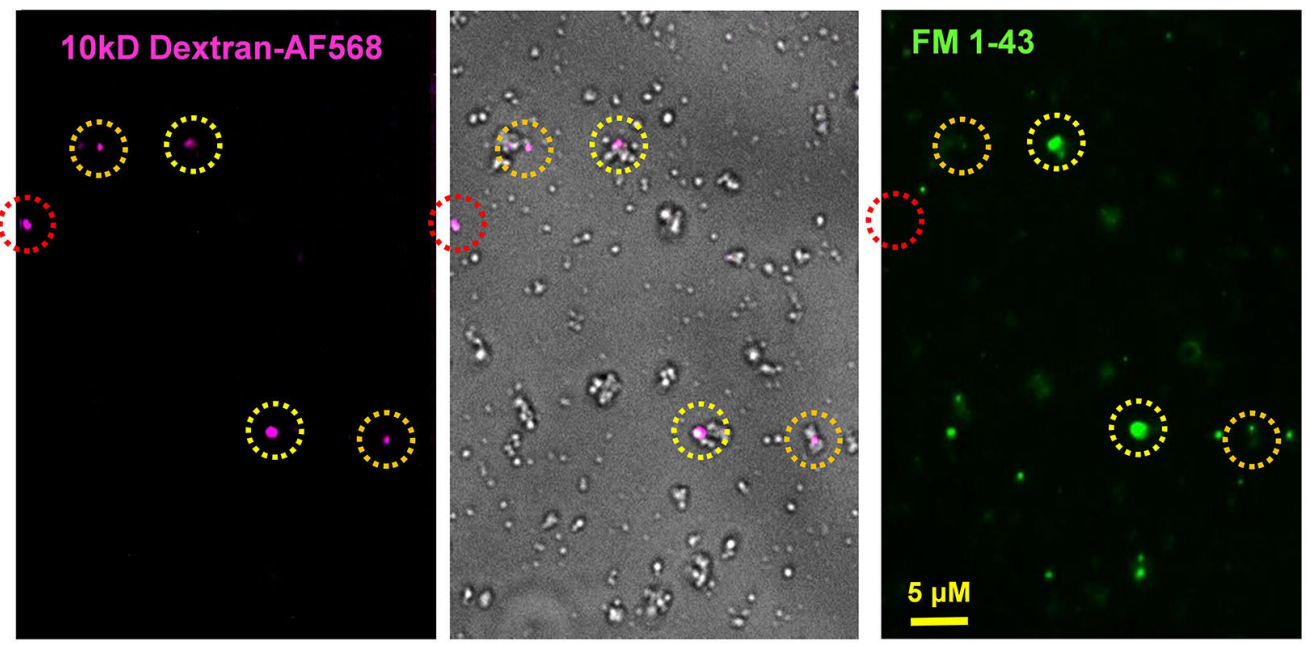

C
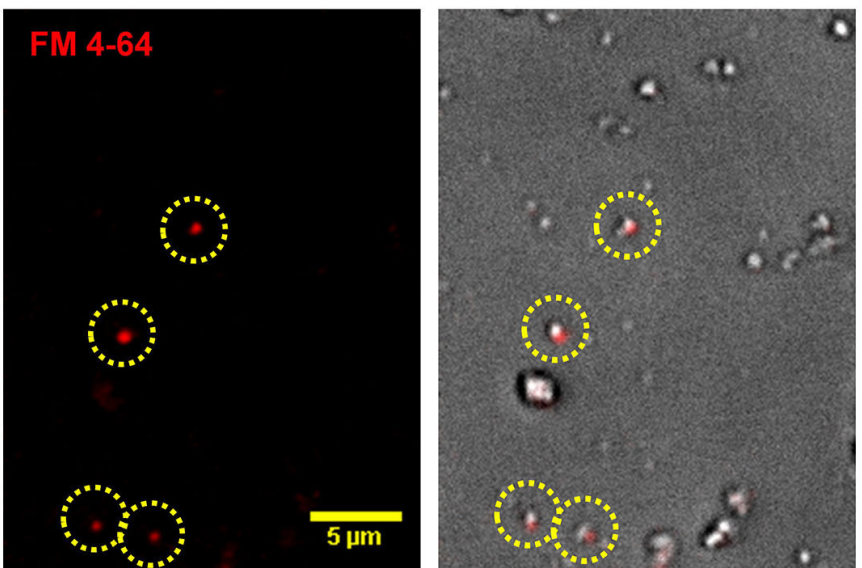

FIGURE 2 | Cryoloaded terminals are capable of SV recycling. (A) Left panel: Electron micrographs of a control presynaptic terminal (PrS) filled with SVs and dense cytoplasm. The active zone (AZ) abuts a scab of postsynaptic membrane (PoS). Right panel: Electron micrograph of a colloidal gold-cryoloaded SSM. Gold particles $\left({ }^{*}\right)$ can be seen within the SSM cytoplasm. See text for methods. (B) Chick synaptosomes cryoloaded with 10 kDa AF568-dextran (left) with a bright-field overlay (center) and FM1-43 loading (right). Cryoloaded SSMs are indicated by dashed circles with robust (yellow), detectable (orange) or absent (red) FM dye staining. In control terminals the majority of cryoloaded SSMs exhibited at least detectable FM dye uptake (see text). (C) Dye uptake is retained through the cryoloading procedure. FM4-64 uptake was performed on fresh SSMs that were then subjected to the freeze-thaw cycle prior to mounting on a coverslip and imaging. The circled SSMs retained styryl dye staining (/eft panel, bright field overlay, right pane/), indicating that recycled SVs survive the cryoloading procedure. and $\mathrm{K}^{+}$depolarization. However, most $(\sim 80 \%$, Figure $3 \mathrm{C})$ of the cryoloaded SSMs exhibited $\mathrm{K}^{+} / \mathrm{Ca}^{2+}$-dependent styryl dye uptake, confirming that the nerve terminals were viable. 


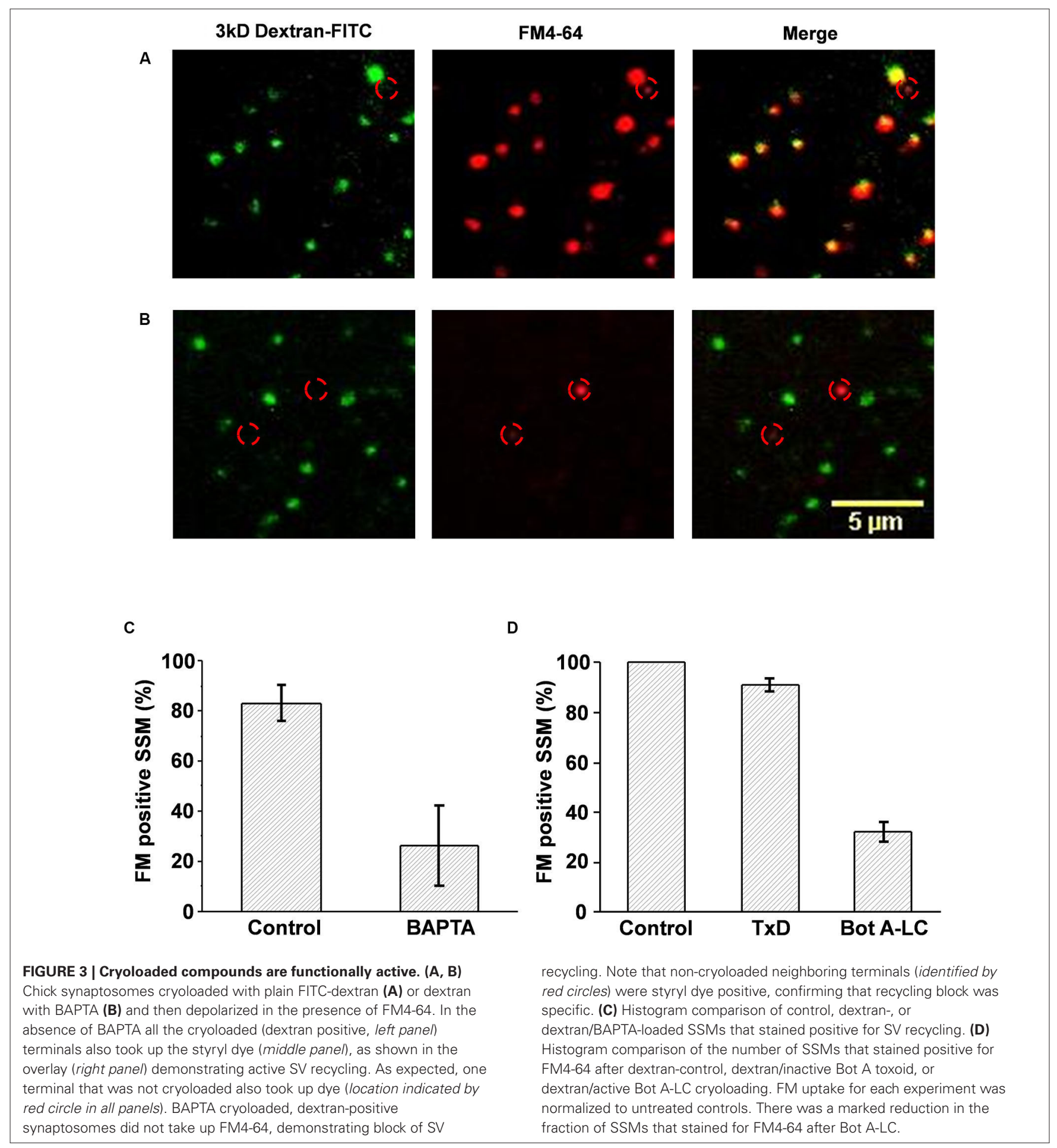

\section{SYNAPTIC VESICLES CAN BE FM LABELED BEFORE CRYOLOADING}

We reasoned that since styryl dyes are internalized within the SV and not the cytoplasm, FM treated SSMs should retain the dye after the freeze-thaw cycle. We confirmed that this was the case: SSMs loaded with FM4-64 (40 $\left.\mathrm{mM} \mathrm{K}^{+}, 5 \mathrm{~min}\right)$ retained the dye after being subjected to the cryoloading procedure (Figure 2C).
This observation is important. FM uptake after cryoloading requires the full SV recycling mechanism and, hence, inhibition could be due to exocytosis, endocytosis or SV transport. The pre-cryoloading FM uptake method permits a test of the effects of intracellular alien compounds that interact solely with the exocytosis arc of the SV cycle. 


\section{CRYOLOADED COMPOUNDS ARE ACTIVE IN THE SYNAPTOSOME CYTOPLASM}

Thus far we have demonstrated that substances can be loaded into the SSM cytoplasm and that the SSM retains its functional capacity to recycle SVs. We next tested if the cryoloaded compounds were biologically active using two membrane-impermeant compounds: BAPTA (Invitrogen), a calcium scavenger and botulinum A toxin light chain (Bot A-LC; Calbiochem), which cleaves a soluble NSF attachment protein receptor (SNARE) protein, SNAP-25, essential for SV exocytosis. Cryoloaded SSMs were identified by co-loading fluorescent dextran, as above.

\section{Block of synaptic vesicle recycling by a cytoplasmic $\mathrm{Ca}^{2+}$ scavenger}

After entering the cytoplasm via the calcium channel, $\mathrm{Ca}^{2+}$ ions diffuse a very short distance before binding to the calcium sensor that gates SV fusion (Stanley, 1993). BAPTA is a membraneimpermeant $\mathrm{Ca}^{2+}$ chelator with a binding "on" rate that is sufficiently fast and has a sufficiently high affinity to capture the ion before it can reach the sensor to block transmission (Adler et al., 1991). FM uptake assays demonstrated a marked inhibition of SV recycling in the BAPTA (30 mM) loaded terminals (26.2 $\pm 16.1 \%$ FM positive SSMs, $n=3$ experiments) compared to controls (carrier alone; $83.1 \pm 7.3 \%, n=3$; $p_{\text {paired } t \text {-test }}<0.05$; Figures $3 B, C$ ). Thus, cryoloaded BAPTA blocked SV recycling and was active.

\section{Block of synaptic vesicle recycling by an intracellular toxin}

The main purpose of the cryoloading method is to introduce large peptides into the terminal. We used botulinum A lightchain (Bot A-LC) as a test. Whole Bot A toxin blocks synaptic transmission by first accessing the nerve terminal cytoplasm and then by cleaving SNAP-25 (Schiavo et al., 1995). These two functions are achieved by separate peptides: the heavy chain creates a pore for membrane penetration allowing cytoplasm access for the light chain $(\sim 50 \mathrm{kDa})$ with the enzymatic activity. Hence, the light chain is biologically inactive unless artificially introduced into the cytoplasm. We cryoloaded Bot A-LC (0.2 $\mu \mathrm{M})$, identifying loaded terminals with co-loaded dextran $3 \mathrm{kDa}$-FITC. This was compared to two controls: dextran alone or dextran with inactivated botulinum A toxoid (TxD; $0.2 \mu \mathrm{M}$; Metabiologics), normalizing to the dextran-alone terminals. While there was a small reduction in the mean fraction of SSMs that were FM loaded in the TxD loaded SSMs, this failed to reach significance $(91.1 \pm$ $2.3 \% ; n=3$ experiments; $p$ paired $t$-test $>0.05)$. In contrast, dye uptake was markedly reduced in the Bot A-LC loaded terminals $\left(32.0 \pm 4.0 \%\right.$; $p$ paired $t$-test, toxoid $<0.01, p_{\text {paired }} t$-test, untreated $<0.01$; Figure 3D). Thus, this experiment confirmed both that it is possible to cryoload large peptides and that these retain their biological activity.

Previous experimental analyses of SV recycling have used three main methods. First, injection of the compounds into presynaptic terminals that have unusually large presynaptic terminals, such as at the squid (Miledi, 1969; Llinas et al., 1981) and lamprey axon giant synapses (Martin and Ringham, 1975; Low et al., 1999), or chick (Stanley, 1989; Stanley and Goping, 1991) and rat (Stanley and Goping, 1991; Forsythe, 1994; Watanabe et al., 2010) calyceal synapses (Chen et al., 2002). While effective, these are generally experimentally challenging and laborious, prone to false negatives and because of their small size, can not be applied to the large spectrum of normal CNS bouton presynaptic terminals. Second, is to make the test compound membrane permeant, such as with the addition of an Acetoxymethyl (AM) group (Tsien, 1981). However, these are limited by the type of compound that can be carried and by possible biological activity of the tag itself, not to mention confidence that loading occurred. The third alternative has been to use genetic means to induce the neuron to express proteins and then assay the outcome using either styryl dyes or evoked postsynaptic electrical responses. This method has advantages that include application to virtually any synapse and also the deletion of existing proteins. However, these are countered by a highly involved and unpredictable preparatory effort; aberrations due to secondary changes during the expression; a requirement for the protein to be transported to the terminal, and often confidence that the intended perturbation has occurred as predicted.

The main advance of our project is the development of a very simple method to reliably introduce virtually any molecule into the cytoplasm of viable identified SSMs. The idea of loading compounds into synaptosomes by freeze-thaw was introduced by Nichols (Nichols et al., 1989; see also: Tamura et al., 2001). Those studies demonstrated calcium-sensitive transmitter efflux, detected as radioisotopes after freeze thaw. However, the reported freeze-thaw methods were not optimized nor tested for efficacy and the bulk-assay method used could not distinguish the actual source of the isotope: whether from actual cryoloaded SSMs, frozen, but unloaded SSMs, or even ruptured SSMs. While we recognize the innovation of our predecessors for the concept, we argue that by monitoring individual SSMs and tagging loaded terminals with a fluorescent marker (dextran), we present the first definitive demonstration of freeze-thaw-loading of active compounds into functional synaptosome presynaptic terminals.

It is reasonable to presume that compounds enter the terminals during cryoloading by bulk flow. Thus, at least for the small compounds, the cytoplasmic concentration should be equal to the extracellular solution concentration. However, future studies will be necessary to calibrate the degree of equilibriation in order to predict the final intracellular concentration. The method permits the introduction of peptides, proteins or markers followed by the evaluation of presynaptic function by fluorescent tagging or other methods and works equally well in other species such as rodent (data not shown). We also report a novel method to identify individual SSMs by their postsynaptic receptor types, making it possible (by post-recycling immunostaining) to correlate SV recycling kinetics with the specific type of SSM. We anticipate that this method will be of particular interest in the exploration of disorders that affect specific neuron types, such as dopaminergic terminals in Parkinson's disease (Picconi et al., 2012) or cholinergic ones in Alzheimer's (Yao et al., 2003). Thus, the cryoloading method opens up the use of the full spectrum of CNS presynaptic terminals for SV recycling analysis and can be carried out with standard fluorescence microscopy. A particular advantage of the method is that once loaded the frozen synaptosomes can be preserved almost indefinitely for future retesting or new experiments. The method can be readily adapted 
to examine terminals in a specific brain region, such as cortex, cerebellum, striatum or basal ganglia, simply by selection of the starting material.

\section{ACKNOWLEDGMENTS}

We are indebted to Dr. Qi Li for assistance with immunostaining, the University of Toronto Cell and Systems Biology EM facility for services and advice on electron microscopy and for equipment within the TWRI Wright cellular imaging facility.

\section{REFERENCES}

Adler, E. M., Augustine, G. J., Duffy, S. N., and Charlton, M. P. (1991). Alien intracellular calcium chelators attenuate neurotransmitter release at the squid giant synapse. J. Neurosci. 11, 1496-1507.

Begley, J. G., Butterfield, D. A., Keller, J. N., Koppal, T., Drake, J., and Mattson, M. P. (1998). Cryopreservation of rat cortical synaptosomes and analysis of glucose and glutamate transporter activities and mitochondrial function. Brain Res. Brain Res. Protoc. 3, 76-82. doi: 10.1016/s1385-299x(98)00024-5

Bouvier, J. A., Charvin, N., Boudier, J. L., Fathallah, M., Tagaya, M., Takahashi, M., et al. (1996). Distribution of components of the SNARE complex in relation to transmitter release sites at the frog neuromuscular junction. Eur. J. Neurosci. 8, 545-552. doi: 10.1111/j.1460-9568.1996.tb01239.x

Chen, S., Zheng, X., Schulze, K. L., Morris, T., Bellen, H., and Stanley, E. F. (2002). Enhancement of presynaptic calcium current by cysteine string protein. J. Physiol. 538, 383-389. doi: 10.1113/jphysiol.2001.013397

Daniel, J. A., Malladi, C. S., Kettle, E., McCluskey, A., and Robinson, P. J. (2012). Analysis of synaptic vesicle endocytosis in synaptosomes by high-content screening. Nat. Protoc. 7, 1439-1455. doi: 10.1038/nprot.2012.070

Forsythe, I. D. (1994). Direct patch recording from identified presynaptic terminals mediating glutamatergic EPSCs in the rat CNS, in vitro. J. Physiol. 479, 381-387.

Gleitz, J., Beile, A., Wilffert, B., and Tegtmeier, F. (1993). Cryopreservation of freshly isolated synaptosomes prepared from the cerebral cortex of rats. J. Neurosci. Methods 47, 191-197. doi: 10.1016/0165-0270(93)90081-2

Juhaszova, M., Church, P., Blaustein, M. P., and Stanley, E. F. (2000). Location of calcium transporters at presynaptic terminals. Eur. J. Neurosci. 12, 839-846. doi: 10.1046/j.1460-9568.2000.00974.x

Khanna, R., Li, Q., Bewersdorf, J., and Stanley, E. F. (2007). The presynaptic CaV2.2 channel-transmitter release site core complex. Eur. J. Neurosci. 26, 547-559. doi: 10.1111/j.1460-9568.2007.05680.x

Li, Q., Lau, A., Morris, T. J., Guo, L., Fordyce, C. B., and Stanley, E. F. (2004). A syntaxin 1, Galpha(o) and N-type calcium channel complex at a presynaptic nerve terminal: analysis by quantitative immunocolocalization. J. Neurosci. 24, 4070-4081. doi: 10.1523/jneurosci.0346-04.2004

Llinas, R. R., Steinberg, I. Z., and Walton, K. (1981). Relationship between presynaptic calcium current and postsynaptic potential in squid giant synapse. Biophys. J. 33, 323-351. doi: 10.1016/s0006-3495(81)84899-0

Low, P., Norlin, T., Risinger, C., Larhammar, D., Pieribone, V. A., Shupliakov, O., et al. (1999). Inhibition of neurotransmitter release in the lamprey reticulospinal synapse by antibody-mediated disruption of SNAP-25 function. Eur. J. Cell Biol. 78, 787-793. doi: 10.1016/s0171-9335(99)80029-6

Martin, A. R., and Ringham, G. L. (1975). Synaptic transfer at a vertebrate central nervous system synapse. J. Physiol. 251, 409-426.
Miledi, R. (1969). Transmitter release induced by injection of calcium ions into nerve terminals. Proc. R. Soc. Lond. B Biol. Sci. 183, 421-425. doi: 10.1098/rspb. 1973.0026

Nichols, R. A., Wu, W. C., Haycock, J. W., and Greengard, P. (1989). Introduction of impermeant molecules into synaptosomes using freeze/thaw permeabilization. J. Neurochem. 52, 521-529. doi: 10.1111/j.1471-4159.1989.tb09151.x

Picconi, B., Piccoli, G., and Calabresi, P. (2012). Synaptic dysfunction in Parkinson's disease. Adv. Exp. Med. Biol. 970, 553-572. doi: 10.1007/978-3-7091-0932-8_24

Schiavo, G., Rossetto, O., Tonello, F., and Montecucco, C. (1995). Intracellular targets and metalloprotease activity of tetanus and botulism neurotoxins. Curr. Top. Microbiol. Immunol. 195, 257-274. doi: 10.1007/978-3-642-85173-5_12

Stanley, E. F. (1989). Calcium currents in a vertebrate presynaptic nerve terminal: the chick ciliary ganglion calyx. Brain Res. 505, 341-345. doi: 10.1016/00068993(89)91465-0

Stanley, E. F. (1993). Single calcium channels and acetylcholine release at a presynaptic nerve terminal. Neuron 11, 1007-1011. doi: 10.1016/08966273(93)90214-c

Stanley, E. F., and Goping, G. (1991). Characterization of a calcium current in a vertebrate cholinergic presynaptic nerve terminal. J. Neurosci. 11, 985-993. doi: 10.1016/0896-6273(91)90371-6

Tamura, Y., Ozkan, E. D., Bole, D. G., and Ueda, T. (2001). IPF, a vesicular uptake inhibitory protein factor, can reduce the $\mathrm{Ca}(2+)$. -dependent, evoked release of glutamate, GABA and serotonin. J. Neurochem. 76, 1153-1164. doi: 10.1046/j. 1471-4159.2001.00120.x

Tsien, R. Y. (1981). A non-disruptive technique for loading calcium buffers and indicators into cells. Nature 290, 527-528. doi: 10.1038/290527a0

Watanabe, H., Yamashita, T., Saitoh, N., Kiyonaka, S., Iwamatsu, A., Campbell, K. P., et al. (2010). Involvement of Ca2+ channel synprint site in synaptic vesicle endocytosis. J. Neurosci. 30, 655-660. doi: 10.1523/jneurosci.3214-09.2010

Whittaker, V. P. (1993). Thirty years of synaptosome research. J. Neurocytol. 22, 735-742. doi: 10.1007/bf01181319

Wong, F. K., Li, Q., and Stanley, E. F. (2013). Synaptic vesicle capture by CaV2.2 calcium channels. Front. Cell. Neurosci. 7:101. doi: 10.3389/fncel.2013.00101

Yao, P. J., Zhu, M., Pyun, E. I., Brooks, A. I., Therianos, S., Meyers, V. E., et al. (2003). Defects in expression of genes related to synaptic vesicle trafficking in frontal cortex of Alzheimer's disease. Neurobiol. Dis. 12, 97-109. doi: 10. 1016/s0969-9961(02)00009-8

Conflict of Interest Statement: The authors declare that the research was conducted in the absence of any commercial or financial relationships that could be construed as a potential conflict of interest.

Received: 29 September 2013; paper pending published: 01 November 2013; accepted: 06 January 2014; published online: 23 January 2014.

Citation: Nath AR, Chen RHC and Stanley EF (2014) Cryoloading: introducing large molecules into live synaptosomes. Front. Cell. Neurosci. 8:4. doi: 10.3389/fncel. 2014.00004

This article was submitted to the journal Frontiers in Cellular Neuroscience.

Copyright (c) 2014 Nath, Chen and Stanley. This is an open-access article distributed under the terms of the Creative Commons Attribution License (CC BY). The use, distribution or reproduction in other forums is permitted, provided the original author(s) or licensor are credited and that the original publication in this journal is cited, in accordance with accepted academic practice. No use, distribution or reproduction is permitted which does not comply with these terms. 